\title{
Correction to: Anti-Müllerian hormone serum levels in systemic lupus erythematosus patients: influence of the disease severity and therapy on the ovarian reserve
}

\section{Clara Di Mario ${ }^{1}$ - Luca Petricca ${ }^{2}$ Maria Rita Gigante ${ }^{2} \cdot$ Angelina Barini $^{3,4} \cdot$ Antonella Barini $^{4} \cdot$ Valentina Varriano $^{1} \cdot$ Annamaria Paglionico ${ }^{1}$ Paola Cattani ${ }^{5,6} \cdot$ Gianfranco Ferraccioli $^{1,2} \cdot$ Barbara Tolusso $^{2} \cdot$ Elisa Gremese $^{1,2}$}

Published online: 1 December 2018

(c) Springer Science+Business Media, LLC, part of Springer Nature 2018

Correction to: Endocrine 2018

https://doi.org/10.1007/s12020-018-1783-1

The original version of this article unfortunately contained a mistake in given and family names of all the authors.

The correct author names are given below:

Clara Di Mario

Luca Petricca

Maria Rita Gigante

Angelina Barini
Antonella Barini

Valentina Varriano

Annamaria Paglionico

Paola Cattani

Gianfranco Ferraccioli

Barbara Tolusso

Elisa Gremese

The original article has been corrected.

The original article can be found online at https://doi.org/10.1007/ s12020-018-1783-1.

Elisa Gremese

elisa.gremese@unicatt.it

1 Institute of Rheumatology, Università Cattolica del Sacro Cuore, Rome, Italy

2 Division of Rheumatology, Fondazione Policlinico Universitario “A. Gemelli"-I.R.C.C.S, Rome, Italy

3 Institute of Biochemistry and Clinical Biochemistry, Università Cattolica del Sacro Cuore, Rome, Italy

4 Institute of Biochemistry and Clinical Biochemistry, Fondazione Policlinico Universitario “A. Gemelli”-I.R.C.C.S, Rome, Italy

5 Institute of Microbiology, Università Cattolica del Sacro Cuore, Rome, Italy

6 Institute of Microbiology, Fondazione Policlinico Universitario “A. Gemelli”-I.R.C.C.S, Rome, Italy 